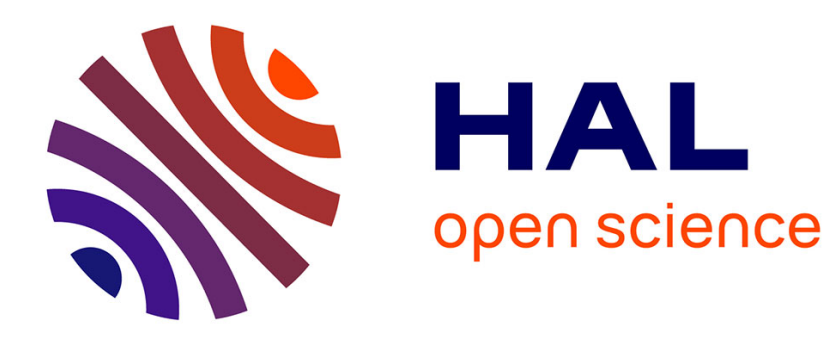

\title{
Genre et histoire: le cas des Mémoires féminins de la Révolution anglaise
}

\author{
Claire Gheeraert-Graffeuille
}

\section{To cite this version:}

Claire Gheeraert-Graffeuille. Genre et histoire: le cas des Mémoires féminins de la Révolution anglaise. Dix-septième siècle, 2012. hal-01961278

\section{HAL Id: hal-01961278}

\section{https://hal-normandie-univ.archives-ouvertes.fr/hal-01961278}

Submitted on 6 Mar 2019

HAL is a multi-disciplinary open access archive for the deposit and dissemination of scientific research documents, whether they are published or not. The documents may come from teaching and research institutions in France or abroad, or from public or private research centers.
L'archive ouverte pluridisciplinaire HAL, est destinée au dépôt et à la diffusion de documents scientifiques de niveau recherche, publiés ou non, émanant des établissements d'enseignement et de recherche français ou étrangers, des laboratoires publics ou privés. 


\section{Genre et histoire : le cas des mémoires féminins de la Révolution anglaise}

Mis à part quelques travaux isolés, en particulier sur les mémoires féminins, les études en langue française sur les femmes et la Révolution anglaise restent rares ${ }^{1}$. En GrandeBretagne, en revanche, le rôle des femmes pendant les années de guerres civiles, sans jamais avoir été au cœur des débats historiographiques, a souvent été remarqué : à la fin du XVII siècle, par exemple, l'historien anglais Edward Hyde mentionne avec mépris les femmes qui pétitionnèrent pour la paix en 1643 ainsi que celle celles qui se mirent à prêcher ${ }^{2}$, tandis qu'au $\mathrm{XIX}^{\mathrm{e}}$ siècle, généalogistes et historiens rééditent de nombreux mémoires féminins ${ }^{3}$. Nous commencerons par examiner les enjeux méthodologiques des études sur les femmes pendant la Révolution anglaise, avant de mesurer l'apport historiographique que représentent quelques mémoires féminins de cette période.

Depuis le début des années 1990, le rôle des femmes pendant la Révolution anglaise, leur participation aux débats politiques et religieux ainsi que leurs écrits de plus en plus nombreux, font l'objet d'articles et d'ouvrages importants ${ }^{4}$. Ces études sont le produit de recherches universitaires dont les premiers jalons ont été posés très tôt. Dès les premières décennies du $\mathrm{XX}^{\mathrm{e}}$ siècle, Ellen A. McArthur exhume les pétitions féminines présentées au long Parlement, tandis que Champlin Burrage et Ethyn Morgan Williams examinent de près

\footnotetext{
${ }^{1}$ Voir François Guizot, «Mistriss Hutchinson », in Études biographiques sur la Révolution d'Angleterre par M. Guizot, Paris, Didier, 1851, pp. 217-256 ; Marie-Madeleine Martinet, «D'aiguille en fil : l'expérience et l'écriture dans les Mémoires de Défaite au XVII ${ }^{\mathrm{e}}$ siècle », Études Anglaises, 1988, n 41, vol. 2, pp. 129-142 ; Ann Hughes, «L'action des femmes dans les révolutions anglaises », in Christiane Fauré (dir.), Paris, PUF, 1997, pp. 162-189 ; Claire Gheeraert-Graffeuille, La Cuisine et le forum. L'émergence des femmes sur la scène publique pendant la Révolution anglaise 1640-1660, Paris, L'Harmattan, « Des idées et des femmes », 2005 ; Stéphane Jettot, «L'Ambassadeur dans sa famille : l'exemple de l'Angleterre sous les derniers Stuart », Dixseptième siècle, 2010, $\mathrm{n}^{\circ}$ 249, en particulier pp. 671-673 (sur Ann, Lady Fanshawe) ; Sandrine Parageau, «Catching "the Genius of the Age": Margaret Cavendish, Historian and Witness », Études Épistémè, 2010, $\mathrm{n}^{\circ} 17$, pp. 55-67.

${ }^{2}$ Edward Hyde, The History of the Rebellion and Civil Wars in England, Dunn Macray (ed.), Oxford, Clarendon Press, 1888, 1969, vol. 3, p. 139 et vol. 2, p. 218.

${ }^{3}$ Par exemple, Memoirs of the Life of Colonel Hutchinson [...] Written by his Widow Lucy, Julius Hutchinson (ed.), London, 1806; Memoirs of Lady Fanshawe, Nicholas Harris Nicolas (ed.), London, 1829; Memoirs of the Duke and Duchess of Newcastle; the Life of William Cavendish, Duke of Newcastle, C. H. Firth (ed.), 1886; The Autobiography of Anne Lady Halkett, John Gough Nichols (ed.), Camden Society, 1875.

${ }^{4}$ Voir en particulier Neil H. Keeble (ed.), The Cambridge Companion to Writing of the English Revolution, Cambridge, Cambridge UP, 2001; Laura Lunger Knoppers (ed.), The Cambridge Companion to Early Modern Women's Writing, Cambridge, Cambridge UP, 2009. Voir aussi Phyllis Mack, Visionary Women: Ecstatic Prophecy in Seventeenth-Century England, Berkeley, University of California Press, 1992; Hilary Hinds, God's Englishwomen: Seventeenth-Century Radical Sectarian Writing and Feminist Criticism, Manchester, Manchester UP, 1996; Jacqueline Eales, Women in Early Modern England, 1500-1700, Abington, Routledge, 1998; Stevie Davies, Unbridled Spirits: Women of the English Revolution, London, The Woman Press, 1998; Mihoko Suzuki, Subordinate Subjects: Gender, The Political Nation and Literary Form in England 1588-1688, Aldershot, Ashgate, 2003; Katharine Gillespie, Domesticity and Dissent in the Seventeenth Century: English Women's Writing and the Public Sphere, Cambridge, Cambridge UP, 2004; Diane Purkiss, Literature, Gender and Politics during the English Civil War, Cambridge UP, 2005; Marcus Nevitt, Women and the Pamphlet Culture of Revolutionary England, Aldershot, Ashgate, 2006 ; Susan Wiseman, Conspiracy and Virtue, Women, Writing and Politics in Seventeenth-Century England, Oxford, Oxford UP, 2006; Kate Chedgzoy, Women's Writing in the British Atlantic World. Memory, Place and History, 1550-1700, Cambridge, Cambridge UP, 2007; Ann Hughes, Gender and the English Revolution, Abingdon, Routledge,2012.
} 
prophéties et prédications féminines ${ }^{5}$. À la fin des années 1950, Keith Thomas propose une nouvelle réflexion sur le rôle des femmes dans les sectes radicales ${ }^{6}$, tandis que Patricia Higgins fournit une étude précieuse des pétitions féminines des années 1640 et 1650, établissant une bibliographie très complète toujours utile à ce jour ${ }^{7}$. Curieusement, pendant les années 1970, malgré le développement des études féministes et le radicalisme ambiant, l'activisme politique et religieux des femmes de la Révolution anglaise attire peu l'attention des universitaires anglo-saxons qui se concentrent davantage sur les $\mathrm{XIX}^{\mathrm{e}}$ et $\mathrm{XX}^{\mathrm{e}}$ siècles, généralement considérés comme le moment d'éclosion du féminisme moderne ${ }^{8}$. Cependant, à partir des années 1980, et encore plus nettement pendant les années 1990, des universitaires, souvent proches des mouvements féministes, redécouvrent pétitions, prophéties, traités et pamphlets laissés par les femmes des années 1640 et 1650. L'ouvrage d'Elaine Hobby sur les écrits féminins au milieu du XVII ${ }^{\mathrm{e}}$ siècle ainsi que la bibliographie compilée par Hilda L. Smith et Susan Cardinale ${ }^{9}$ sont à cet égard exemplaires : en sortant de l'obscurité les nombreux textes publiés par les femmes au milieu du XVII ${ }^{\mathrm{e}}$ siècle, elles ouvrent les études sur la Révolution aux historiens de la littérature. Parallèlement, des travaux sur le radicalisme politique et religieux mettent en évidence le rôle important joué par les femmes dans les sectes dissidentes. Ainsi, l'ouvrage de Christopher Hill, The World Turned Upside Down, qui consacre un chapitre aux mœurs sexuelles et au statut de la femme dans les sectes radicales, est suivi d'analyses plus documentées, comme celles de Richard L. Greaves, de Claire Cross, de Patricia Ludlow ${ }^{10}$.

Ces études pionnières, de sensibilité souvent marxiste ou féministe, seront relues et nuancées à la fin des années 1980 à la lumière de la catégorie du genre qui renvoie à la relation sociale et culturelle entre les sexes. C'est le rapport hommes-femmes et non plus la nature des femmes ou leur histoire en tant que groupe social autonome qui intéressent les chercheurs $^{11}$. En outre, le genre se prête particulièrement bien à l'historicisation, car contrairement au sexe plus biologique, il est modelé par les changements culturels, sociaux et historiques. Dans ce sens, contrairement à ce qu'ont pu laisser entendre certaines féministes,

\footnotetext{
${ }^{5}$ Ellen A. McArthur, «Women Petitioners and the Long Parliament », English Historical Review, 1908, n ${ }^{\circ} 24$, pp. 698-709; Champlin Burrage, «Anna Trapnel's Prophecies », English Historical Review, 1911, $\mathrm{n}^{\circ} 26$, pp. 526-542; Ethyn Morgan Williams, «Women Preachers in the Civil War », Journal of Modern History, 1929, $\mathrm{n}^{\circ} 1$, pp. 561-569.

${ }_{7}^{6}$ Keith Thomas, «Women and the Civil War Sects », Past and Present, 1958, $\mathrm{n}^{\circ}$ 13, pp. 42-62.

${ }^{7}$ Patricia Higgins, «The Reactions of Women with Several References to Women Petitioners », Politics, Religion and the English Civil War, Brian Manning (ed.), London, Edward Arnold, 1973, pp. 178-220.

${ }^{8}$ Voir cependant Christine Berg and Philippa Berry, " "Spiritual Whoredom" : An Essay on Female Prophets in the Seventeenth Century », in Francis Barker et al. (eds.), $1642:$ Literature and Power in the Seventeenth Century, Colchester, University of Essex, 1981, pp. 37-54; Margaret George, Women in the First Capitalist Society, Brighton, The Harvester Press, 1988.

${ }_{9}^{9}$ Voir Elaine Hobby, Virtue of Necessity. English Women's Writing 1649-1688, Ann Arbor, University of Michigan, 1988 et Hilda Smith and Susan Cardinal (eds.), Women and the Literature of the Seventeenth Century. An Annotated Bibliography based on Wing's Short-Title Catalogue, Westport, Connecticut, Greenwood Press, 1990.

${ }^{10}$ Voir Christopher Hill, The World Turned Upside Down. Radical Ideas during the English Revolution, London, Penguin, 1972, 1991, pp. 306-323; Claire Cross, " "He-Goats before the Flocks": A Note on the Part Played by Women in the Founding of Some Civil War Churches », Studies in Church History, 1972, n 8, pp. 195-202; Richard L. Greaves, «The Role of Women in Early English Nonconformity », Church History, 1983, n 52, pp. 299-311; Dorothy P. Ludlow, « Shaking Patriarchy's Foundations: Sectarian Women in England 1641-1700 », in Richard L. Greaves (ed.), Triumph over Silence: Women in Protestant History, Westport, Greenwood Press, 1985, pp. 92-123.

${ }^{11}$ Voir Joan W. Scott, « Gender: A Useful Category of Historical Analysis », American Historical Review, 1986, vol. 91, n 5, pp. 1053-1054 ; Denise Riley, "Am I that Name?": Feminism and the Category of "Women" in History, Basingstoke, Macmillan, 1988.
} 
le patriarcat n'est ni monolithique ni éternel ${ }^{12}$; c'est une structure qui fluctue en fonction des représentations mentales d'une époque, des auteurs, ainsi que des événements historiques. La Révolution anglaise est un moment-clé dans l'histoire du genre, comme le note l'historien Anthony Fletcher : «La crise de la guerre civile anglaise et ses conséquences radicales n'est pas une simple crise politique. Elle a fondamentalement ébranlé chez les Anglais la conviction de pouvoir contrôler l'ordre social et l'ordre des sexes ${ }^{13}$. »

Il n'est nullement question ici de mettre en cause cette vision très juste du rapport hommes-femmes pendant la Révolution anglaise. Mais l'on voudrait avancer une hypothèse qui prolonge le raisonnement d'Anthony Fletcher : si, entre 1640 et 1660, un certain nombre de femmes sont sorties du rôle que leur attribuait la société traditionnelle, c'est parce que la conception du genre à l'époque moderne l'autorisait. Au milieu du XVII ${ }^{\mathrm{e}}$ siècle, malgré les avancées médicales, la hiérarchie entre les sexes reste largement déterminée par une vision humorale (et non encore anatomique) dans laquelle la frontière entre le masculin et le féminin n'est pas aussi nette que ne l'auraient souhaité les tenants de la société patriarcale. À cette relative indétermination humorale du genre, correspond, comme l'a magistralement montré Thomas Laqueur, un «modèle unisexe », fondé sur des homologies anatomiques ${ }^{14}$; au milieu du XVII ${ }^{\mathrm{e}}$ siècle : le masculin et le féminin ne constituent pas deux pôles séparés - comme ce sera le cas au XIX $^{\mathrm{e}}$ siècle - mais se situent encore sur un continuum ${ }^{15}$.

Ces représentations mentales du genre ne fournissent pas seulement un cadre à l'activisme des femmes pendant la Révolution anglaise ; elles permettent aussi de comprendre la relative mixité qui prévaut encore au milieu du XVII ${ }^{\mathrm{e}}$ siècle ${ }^{16}$. On voudrait montrer, dans la suite de l'article, que cette fluidité entre les sexes, perceptible tant au niveau des représentations que des rôles inédits joués par les femmes est aussi présente dans l'historiographie de la Révolution anglaise. En particulier, on souhaiterait lire les Mémoires féminins dans la continuité des Mémoires masculins, comme relevant d'une histoire mixte ${ }^{17}$, et non d'une histoire des femmes pour les femmes, que l'on appelle parfois « her-story » (par opposition à «his-story ») et qui prend pour acquis que les femmes, cantonnées dans l'espace domestique et privées d'une éducation classique, auraient forcément recours à d'autres formes d'écriture que les hommes pour raconter leur passé ${ }^{18}$. En envisageant ces textes sous l'angle

\footnotetext{
${ }^{12}$ Sheila Rowbotham, Hidden from History: Three Hundred Years of Women's Oppression and Fight against It, 1973, London, Pluto Press, 1993.

${ }^{13}$ Anthony Fletcher, Gender, Sex and Subordination in England 1500-1800, New Haven, Yale UP, 1995, p. 283.

${ }^{14}$ Thomas Laqueur, Making Sex: Body and Gender from the Greeks to Freud, Cambridge, MA, Harvard UP, 1990, traduit sous le titre La Fabrique du sexe, trad. Michel Gautier, Paris, Gallimard, 1992.

${ }_{15}$ A. Fletcher, Gender, Sex..., op. cit., p. 34.

16 Voir C. Gheeraert-Graffeuille, «Leveller Women Petitioners and the Rhetoric of Power in the English Revolution (1640-1660)», Anglophonia, 2010, n² 27, pp. 15-26.

${ }^{17}$ Sur cette idée que les femmes seraient des historiennes à part entière, voir Devoney Looser, British Women Writers and the Writing of History, 1670-1820, Baltimore and London, The Johns Hopkins UP, 2000, pp. 1-27. Sur les femmes et l'histoire, voire l'article fondateur de Natalie Zemon Davis, « Gender and Genre : Women as Historical Writers, 1400-1820», in Patricia H. Labalme (ed.), Beyond their Sex: Learned Women of the European Past, New York, New York UP, 1980, p. 153-182

${ }^{18}$ Voir Megan Matchinske, Women Writing History in Early Modern England, Cambridge, Cambridge UP, 2009, p. 6: «Because women operated at some remove from traditional historical discussions even before history's entry into disciplinary circles and because the written record documenting that past did not address their lives or their dreams, their need to look to other forms and different historical experiences was both highly fraught and highly sought. » Pour ce genre d'approche voir Cadman Sharon Seelig, Autobiography and Gender in Early Modern Literarture: Reading Women's Lives, 1600-1680, Cambridge, Cambridge UP, 2009.
} 
du genre et de la rhétorique ${ }^{19}$ - sur le modèle de nombreuses études qui portent sur le domaine français ${ }^{20}$ - nous voudrions montrer que les femmes contribuent, à leur manière, à l'écriture de l'histoire de la Révolution anglaise. On s'appuiera sur les écrits de Margaret Cavendish (1623-1673), d'Anne Halkett (1622-1699) et d'Ann Fanshawe (1625-1680), trois royalistes qui défendirent infatigablement la cause de la monarchie ${ }^{21}$. Certes, l'écriture des Mémoires n'est pas le privilège des défenseurs du roi - les Mémoires les plus connus et les plus commentés, ceux de Lucy Hutchinson, défendent la cause républicaine - mais dans l'espace de cet article le choix d'un corpus cohérent sur le plan idéologique facilitera les comparaisons thématiques et formelles.

$\mathrm{Au} \mathrm{XVII}^{\mathrm{e}}$ siècle, le terme de «Mémoires » est moins utilisé en Angleterre qu'en France $^{22}$, les Anglais du XVII ${ }^{\mathrm{e}}$ siècle ayant tendance à l'appliquer davantage aux textes français du Grand Siècle qu'à ceux de leurs compatriotes ${ }^{23}$. Cependant, critiques et historiens s'accordent à dire que les Mémoires constituent «l'un des genres dominants dans lequel a été écrite la Guerre Civile, de 1660 jusqu'à la publication de L'Histoire pionnière de Catherine Macaulay à la fin du XVIIIe siècle »; ce genre, ajoute Susan Wiseman, est «central dans la construction du monde politique de la Restauration et sa compréhension de la guerre civile anglaise. $^{24} \gg$ Les auteurs que nous allons évoquer, qui écrivent au moment de la Restauration, n'emploient pas le terme de Mémoires, contrairement à leurs éditeurs des $\mathrm{XIX}^{\mathrm{e}}$ et $\mathrm{XX}^{\mathrm{e}}$ siècles ${ }^{25}$. Cependant leurs récits rentrent parfaitement dans les définitions de Furetière et de l'Oxford English Dictionary.

D'après le Dictionnaire universel de Furetière et l'Oxford English Dictionary, les Mémoires sont des textes d'histoire, écrits par des individus qui ont une connaissance particulière des événements qu'ils racontent ; autrement dit, ce sont des récits à la croisée de l'histoire particulière et de l'histoire générale : « des Livres d'Historiens, écrits par ceux qui ont eu part aux affaires ou qui en ont esté tesmoins oculaires, ou qui contiennent leur vie ou leurs principales actions: Ce qui répond à ce que les Latins appelloient commentaires. » On retrouve une définition similaire dans les entrées 3a et 3b de l'Oxford English Dictionary:

\footnotetext{
${ }^{19}$ C'est un aspect négligé par la critique anglo-saxonne, ce que semblent regretter Kevin Sharpe et Steven N. Zwicker (eds.), Writing Lives: Biography and Textuality, Identity and Representation in Early Modern England, Oxford, Oxford UP, 2008, p. 6: «We need, that is, a deeper sense of the rhetoric of early modern life. »

${ }^{20}$ Voir en particulier Frédéric Charbonneau, Les Silences de l'Histoire. Les Mémoires français du XVII siècle, Laval, Presse de l'Université de Laval, 2001 ; Emmanuèle Lesne, La Poétique des Mémoires (1650-1685), Paris, Honoré Champion, 1996 ; Marc Fumaroli, «Les Mémoires au carrefour des genres en prose », La Diplomatie de l'esprit, Paris, Herman, 1994, pp. 183-215; Frédéric Briot, Usage du monde, usage de soi. Enquête sur les Mémorialistes d'Ancien régime, Paris, Le Seuil, 1991.

${ }^{21}$ The Memoirs of the Life of Colonel Hutchinson relèvent de l'histoire comme nous l'avons montré dans «L'atelier de l'historienne : "The Life of John Hutchinson” », Études Épistémè, 2010, n 17, pp. 69-86.

${ }^{22}$ Voir E. Lesne, La Poétique des Mémoires, op. cit. p. 21. Voir Gilbert Burnet, The Memoires of the Lives and Actions of James and William Dukes of Hamilton and Castleherald, London, 1677, «Preface », sig. [av]: « Of all Nations in the world the French have delighted most in these Writings, so that the Memoires, written in that Nation and Language since the day of Henry 3d, would also make up a Library: and every year we get over new Memoires of some one Great Person or another. [...] this way of Writing tales now more in the World than any sort of History ever did. »

${ }^{23}$ F. Briot (Usage du monde, op. cit. p. 25) note qu'en France le terme de «Mémoires » n'est pas toujours revendiqué par les mémorialistes du XVII ${ }^{\mathrm{e}}$ siècle.

${ }^{24}$ S. Wiseman, Conspiracy and Virtue, op. cit., p. 316. Voir aussi Ian Donaldson, « National Biography and the Arts of Memory », in Mapping Lives: The Uses of Biography, Oxford, Oxford UP, 2002, pp. 67-68.

${ }^{25}$ The Memoirs of Anne, Lady Halkett and Ann, Lady Fanshawe, John Loftis (ed.), Oxford, Clarendon, 1979 ; The Memoirs of the Duke and Duchess of Newcastle; the Life of William Cavendish, Duke of Newcastle, C. H. Firth (ed.), London,1886 and The Memoirs of Lady Fanshawe, Nicholas Harris Nicolas (ed.), London, London, 1829 ?
} 
(a) une relation qui ne se présente pas comme une histoire complète, mais dont la matière provient d'une connaissance personnelle des événements ou bien de sources particulières d'information.

(b) un récit personnel qui consigne des événements vécus, les personnes rencontrées, les principales actions et transactions auxquelles son auteur a été mêlé. Un récit autobiographique ${ }^{26}$.

La relative rareté du terme Mémoires en Angleterre peut s'expliquer par la faveur dont jouit alors un autre genre, celui de la $«$ Vie ${ }^{27}$, un genre dont relèvent aussi les trois récits que nous évoquons $\mathrm{ici}^{28}$. Margaret Cavendish écrit la «Vie» de William Cavendish ${ }^{29}$, Anne Halkett présente son récit comme «un véritable récit de sa vie ${ }^{30}$, tandis que l'éditeur de 1829 des Mémoires d'Ann Fanshawe, Nicholas Harris Nicolas, la présente comme «l'aimable historienne de la vie de son mari et de la sienne ${ }^{31} »$. Cette appartenance générique n'est pas fortuite. Comme les Mémoires, en effet, le genre de la "Vie » relève à l'époque moderne de l'histoire; dans la typologie de Francis Bacon, c'est l'une des formes que prend l' «histoire parfaite », à côté des «chroniques» et des « relations ${ }^{32}$, une classification à laquelle renvoie Margaret Cavendish dans The Life of William Cavendish ${ }^{33}$, lorsqu'elle affirme que le portrait qu'elle va livrer de son mari rendra l' "Histoire de sa Vie plus parfaite et plus complète ${ }^{34}$. De fait, pour Margaret Cavendish, il va de soi qu'en écrivant la Vie de son époux, elle se conduit en historienne ${ }^{35}:$ «Puisque mon intention principale dans le présent ouvrage est de décrire la Vie et les Actions de mon Noble Seigneur et Mari, William, duc de Newcastle, je le ferai avec autant de Brièveté, de Clarté et de Vérité, qu'on en exige d'un Historien Impartial. ${ }^{36}$ » Selon elle, contrairement à ce que ses détracteurs ne manqueront pas de remarquer, cette posture n'est pas usurpée. En tant que témoin d'événements historiques qui l'ont touchée de près, elle estime que le titre d'historienne lui revient de droit :

\footnotetext{
${ }^{26}$ Oxford English Dictionary Second Edition on CD-ROM (v. 4.0), Oxford UP, 2009.

${ }^{27}$ Voir K. Sharpe et N. Zwicker (eds.), Writing Lives, op. cit., p. 14: «Even after military contest in civil war was subdued by the temporary stabilities of Restoration, life-writing remained central to continuing polemical warfare. » Sur le genre de la vie et la biographie, voir Judith H. Anderson, Biographical Truth. The Representation of Historical Persons in Tudor-Stuart Writing, New Haven, Yale UP, 1984; Thomas F. Mayer and D. R. Woolf (eds.), The Rhetorics of Life-Writing in Early Modern Europe, Ann Arbor, The University of Michigan Press, 1995; Allan Pritchard, English Biography in the Seventeenth Century. A Critical Survey, Toronto, Toronto UP, 2005.

${ }^{28}$ F. Briot (op. cit., p. 25) remarque que ce terme de «Mémoires » n'est pas non plus revendiqué par tous les « mémorialistes » français.

${ }^{29}$ Margaret Cavendish, The Life of the Thrice Noble, High and Puissant Prince William Cavendish, London, 1667.

${ }^{30}$ Voir http://web.warwick.ac.uk/english/perdita/html/ms_NLS6494.htm consulté le 12 septembre 2012 pour des extraits des Meditations du 22 avril 1678.

${ }^{31}$ Voir N. Harris Nicolas (ed.), Memoirs of Lady Fanshawe, op. cit., p. xiii : «the amiable historian of the lives of her husband and herself $»$.

${ }^{32}$ Francis Bacon, The tvvoo bookes of Francis Bacon. Of the proficience and aduancement of learning, diuine and humane, London, 1605, $2^{\text {nd }}$ book, p. $10:$ : HISTORY which may be called IVST and PARFITE Historie, is of therre kinds, according to the object which it propoundeth, or pretendeth to represent: for it either representeth a TIME, or a PERSON, or an ACTION. The first we call CHRONICLES, the second LIVES, and the third NARRATIONS, or RELATIONS. Of these although the first bee the most compleate and asbolute kinde of Historie, and hath more estimation and glory : yet the second excelleth it in profit and use, and the third in veritie \& sinceritie. »

${ }^{33}$ Margaret Cavendish était une grande lectrice de Bacon. Voir Sandrine Parageau, Les Ruses de l'ignorance. La contribution des femmes à l'avènement de la science moderne en Angleterre, Paris, Presses de la Sorbonne Nouvelle, 2010, pp. 96-99.

${ }^{34}$ M. Cavendish, The Life ..., op. cit., p. 103: «to render the History of his Life more perfect and compleat. » Voir . Bacon, op. cit., p. 10.

${ }^{35}$ Voir S. Parageau, « Catching "the Genius of the Age" : Margaret Cavendish, Historian and Witness », art. cit.

${ }^{36}$ M. Cavendish, The Life..., op. cit., sig. B: «Since my chief intent in this present Work, is to describe the Life and Actions of My Noble Lord and Husband, William, Duke of Newcastle, I shall do it with as much Brevity, Perspicuity and Truth, as is required of an Impartial Historian. »
} 
Il n'existe pas de contradiction entre le fait que je sois une femme et le fait que j'écrive à propos de guerres qui n'ont vu s'affronter ni les Mèdes et les Perses, ni les Grecs et les Troyens, ni les Chrétiens et les Turcs, mais mes propres Concitoyens, dont je connais bien les Coutumes et les Inclinations, ainsi que la plupart des militaires qui occupent des positions considérables dans l'armée; et, en plus de tout cela - et c'est le plus important - mon Noble et Loyal Seigneur a joué le rôle principal dans cette fatale tragédie ${ }^{37}$.

En insistant ici sur la notion de témoignage et en passant sous silence les atouts que peuvent représenter pour un historien la maîtrise de l'éloquence et une connaissance du passé ${ }^{38}$, Margaret Cavendish rejoint non seulement les mémorialistes français mais aussi la plupart des historiens de la Révolution anglaise, selon lesquels, en ces temps de division, seuls les historiens témoins peuvent prétendre accéder à la vérité. Pour reprendre les mots du modéré Thomas Fuller (1608-1661), « les histoires les plus instructives et celles auxquelles les esprits sages accordent le plus de prix sont celles qui furent écrites par les témoins oculaires des événements, comme Thucydide, qui rapporte la guerre du Péloponnèse. ${ }^{39}$ Comme nombre de ses contemporains, la duchesse de Newcastle reconnaît ainsi plus de valeur à l'histoire particulière, écrite par un témoin, qu'à l'histoire générale, car l'histoire particulière «tourne sur son Axe, et la plupart du temps, demeure dans la circonférence de la Vérité ${ }^{40} »$; l' «histoire nationale», en revanche, est source de conflits et «ravive les anciennes querelles $\gg{ }^{41}$. Ce commentaire convient parfaitement aux récits de nos trois mémorialistes. En rapportant des destins particuliers, le leur et celui de leur famille, celles-ci donnent à lire une histoire «vraie » de la Révolution, souvent en décalage avec l'histoire officielle défendue par la monarchie. Leurs écrits méritent donc d'être étudiés pour eux-mêmes et non comme de simples sources utiles à l'histoire militaire de la période ${ }^{42}$.

Margaret Cavendish défend la vérité de ses Mémoires en les présentant comme un témoignage ; cependant, parmi les trois récits étudiés ici, The Life of [...] William Cavendish est paradoxalement celui qui procure le témoignage le moins direct. Ainsi, le premier Livre (qui porte sur la vie de William avant qu'il n'épouse Margaret) est fondé non sur le témoignage de la duchesse, mais sur les dires d'un certain Mr Rolleston, le secrétaire de son

\footnotetext{
${ }^{37}$ M. Cavendish, The Life ..., op. cit. [sig. $\mathrm{c}^{\mathrm{v}}$ ]: « [It is not] inconsistent with my being a Woman, to write of Wars, that was neither between Medes and Persians, Greeks and Trojans, Christians and Turks; but among my own Countreymen, whose Customs and Inclinations, and most of the Persons that held any considerable Place in the Armies, was well known to me; and besides all that (which is above all) my Noble and Loyal Lord did act a chief Part in that fatal Tragedy, to have defended [...] his most Gracious Soveraign, from the fury of his Rebellious Subjects. »

${ }^{38}$ M. Cavendish, The Life..., «The Preface », op. cit., non paginée : «I will [not] endevavour to make show of Eloquence, making Speeches that never was spoken.»Sur le refus de l'éloquence dans les Mémoires et l'autorité du témoin, voir M. Fumaroli, «Les Mémoires, ou l'historiographie royale en procès », in $L a$ Diplomatie de l'esprit de Montaigne à La Fontaine, Paris, 1998, pp. 217-246.

${ }^{39}$ Thomas Fuller, The Church of History of Britain, London, 1656, Livre X, sig. Ggg2v: « the most Informative Histories to Posterity and such as are most highly prized by the judicious, are such as were written by the Eyewitnesses thereof, as Thucydides, the reporter of the Peloponnesian Warre». Sur cette question d'une histoire polémique, voir C. Gheeraert-Graffeuille, «Entre polémique et histoire : comment écrire les guerres civiles anglaises (1640-1660) », in Emmanuel Dupraz et C. Gheeraert-Graffeuille (dir.), La Guerre Civile, Rouen, PURH, à paraître.

${ }^{40}$ M. Cavendish, The Life..., sig. (c) : « [...] the History of the life and action of some particular Person [...] goes not out of its own Circle, but turns on its own Axis, and for the most part keeps within the Circumference of Truth. »

${ }^{41}$ Ibid.

${ }^{42}$ Sur cette question, voir Joanne H. Wright, « Not Just Dutiful Wives and Besotted Ladies: Epistemic Agency in the War Writing of Brilliana Harley and Margaret Cavendish », Early Modern Women: An Interdisciplinary

Journal, 2008, n 4 , p. 1-25.
} 
mari, qui a suivi ce dernier dans toutes ses campagnes militaires, « un témoin oculaire » «dont l'honnêteté et la valeur sont incontestables ${ }^{43}$ ». Dans le deuxième Livre (qui se concentre sur la période allant du mariage des Cavendish jusqu'à leur retour en Angleterre à la Restauration), ce n'est pas tant Margaret qui a le statut de témoin que William, dont elle se contente en quelque sorte de relayer les paroles et les actions. Enfin, dans les troisième et quatrième livres c'est surtout l'inclusion de documents de première main - des lettres du roi, des listes de loyers, etc. - qui servent de caution au témoignage.

Mais il ne faut pas s'y méprendre, cette rigueur historiographique n'est pas gratuite : elle sert à réhabiliter devant ses contemporains William Cavendish, à qui l'on reproche la défaite royaliste à Marston Moor (1644) et son exil sur le Continent. La stratégie de Margaret est double. D'une part, en reconstituant l'histoire de son mari, elle justifie sa conduite, affirmant par exemple que si le duc de Newcastle a fui l'Angleterre, ce n'est pas par lâcheté, mais parce qu'il pressentait que «le Parti du Roi avait perdu, non seulement en Angleterre, mais aussi en Écosse et en Irlande, et qu'il était impossible de résister aux rebelles après la chute fatale de son armée ${ }^{44}$. D'autre part, elle offre une vision désenchantée - voire critique - de la cour et de l'armée, assurant par exemple que William «avait de grand ennemis parmi les proches du roi, de même qu'il avait des ennemis avérés sur le champ de bataille qui essayaient par tous les moyens de le détruire », et qu'il y avait en outre «tromperie, traîtrise et mensonge dans sa propre armée ${ }^{45}$. On ne peut ici que s'étonner de l'audace paradoxale de la duchesse qui cherche à s'attirer les faveurs d'un régime tout en le critiquant.

Dans une certaine mesure, on retrouve la même audace sous la plume d'Anne Halkett et d'Ann Fanshawe qui écrivent aussi pour se justifier à la fois devant leurs descendants et devant la postérité. Certes, leurs Mémoires ne sont pas publiés de leur vivant, mais on perçoit dans leur témoignage la même critique de la monarchie et le même désir de corriger l'histoire officielle qui prévaut à la Restauration ${ }^{46}$.

Dès le début de son récit, Ann Fanshawe se présente comme un témoin, une observatrice de tout ce qui l'entoure. Le fait qu'elle soit très proche de la cour - son mari Richard Fanshawe est alors secrétaire à la guerre du roi Charles $\mathrm{I}^{\mathrm{er}}$ - explique sa position privilégiée : «nous entendions en permanence parler des villes que nous avions perdues et de celles que nous avions gardées; à la fenêtre le triste spectacle de la guerre ${ }^{47}$ ». Mais, en tant que femme et aristocrate, Ann Fanshawe se tient loin des champs de bataille; ce qui l'intéresse ce n'est pas tant la stratégie militaire que l'effet des guerres civiles sur elle-même et sur ses proches. Ainsi, elle ne dit rien de la bataille de Worcester, choisissant de se concentrer plutôt sur les conséquences humaines de cet événement, brouillant la frontière entre histoire nationale et histoire particulière : «Le 2 septembre suivant, eut lieu la bataille de Worcester. Le roi étant porté disparu et n'ayant aucune nouvelle de ton père mort ou vivant

\footnotetext{
${ }^{43}$ W. Cavendish, The Life..., sig. $\left(\mathrm{d}^{\mathrm{v}}\right)$ : « an Eye-witness thereof », « his honesty and worth is unquestionable ».

${ }^{44}$ M. Cavendish, The Life..., op. cit., «Preface »: «Before I end this Preface, I do beseech my Readers not to mistake me when I speak of my Lord's Banishment, as if I would conceal that he went voluntarily out of his Native Country; for it is mots true, that his Lordship prudently perceiving all the King's Party lost, not onely in England, but also in Scotland and Ireland; and that it was impossible to withstand the Rebels, after the fatal overthrow of his Army. »

${ }^{45}$ M. Cavendish, The Life..., p. 118: «My Lord (as heretofore mentioned) had as great private Enemies about his Majesty, as he had public Enemies in the Field, who used all the endeavour they could to pull him down. [...] There was such Jugling, Treachery, and Falshood in his own Army, that it was impossible for my Lord to be prosperous and successful in his Designs and Undertakings. »

${ }^{46}$ Les citations aux Mémoires de Fanshawe et d'Halkett sont tirées de The Memoirs of Anne, Lady Halkett and Ann, Lady Fanshawe, John Loftis (ed.), Oxford, Clarendon Press, 1979.

${ }^{47}$ A. Fanshawe, The Memoirs..., op. cit., p. 111: «We had the perpetuall discourse of losing and gaining of towns and men; at the windows the sad spectacle of war...»
} 
pendant 3 jours, mon affliction était inexpressible ${ }^{48}$. » Mais que l'on ne s'y trompe pas : Ann Fanshawe n'est pas l'auteur naïf qu'on a voulu en faire. Son témoignage, qui s'apparente à un récit d'aventures, est aussi un moyen pour la mémorialiste de défendre son mari et de régler ses comptes avec ses contemporains, une intention qu'elle affiche dès le début de ses mémoires lorsqu'elle affirme : «C'est ma vie et la nécessité, non le plaisir de la vengeance, qui m'ont fait insérer des passages qui feront tantôt apparaître ceux qu'ils concernent sous un jour peu avantageux, tantôt les couvriront d'éloge ${ }^{49}$. » L'une des cibles d'Anne Fanshawe n'est autre que le royaliste Edward Hyde, ministre de Charles $\mathrm{I}^{\mathrm{er}}$ et de Charles II, qu'elle considère responsable de de la disgrâce dans laquelle tomba Sir Richard à la Restauration. Dans la troisième partie de ses mémoires, sa critique se fait plus sévère encore puisqu'elle porte directement sur la politique de Charles II ${ }^{50 .}$

Comme Ann Fanshawe et Margaret Cavendish, Anne Halkett prend soin de ne rapporter que des faits dont elle a été le témoin - lorsqu'elle s'écarte de cette forme de relation, elle s'en justifie auprès du lecteur. Ainsi par exemple, lorsqu'elle raconte l'évasion rocambolesque du duc d'York en avril en 1648 - à laquelle elle a activement participé en lui fournissant des vêtements de fille - elle mentionne à quel moment précis les informations qu'elle fournit lui ont été dictées par un tiers, «Mr N. » qui était «témoins de tout ${ }^{51}$ ». Ailleurs, pour donner plus de véracité à son récit, elle retranscrit les discours qu'elle a entendus au discours direct. C'est le cas, par exemple, lorsqu'elle met en scène sa conversation avec les officiers parlementaires à Fyvie après la bataille de Dunbar (3 septembre 1650), lors de son séjour chez la comtesse de Dunfermline.

- Comment se fait-il (dis-je) que vous avez pris le pouvoir au Parlement et aux différentes formes de gouvernement qui ont suivi puisque c'était le roi que vous vouliez?

- Parce que, dit-il, nous avons découvert très rapidement qu'ils étaient aussi mauvais que lui, et c'est pourquoi nous en avons changé $e^{52}$.

Mais comme dans les cas précédents, le témoignage d'Anne Halkett n'est pas innocent. Certes, à toutes les pages, elle montre sa loyauté à la famille royale. C'est le cas par exemple lorsque le futur roi Charles II, après la défaite de Dunbar, la remercie pour les services qu'elle a rendus à la monarchie :

[Le roi] s'approcha de moi et me dit: «Mrs Murray, j'ai honte de ne pas vous avoir parlé plus tôt, mais la raison en est que je ne pouvais vous remercier suffisamment pour le service que vous avez rendu à mon frère. Mais si un jour je dispose du droit de commander qui est le mien, je ferai tout ce qui est en mon pouvoir pour vous aider. En prononçant ces mots, le roi posa sa main sur les miennes qui se trouvaient sur ma poitrine. Je me suis humblement inclinée et j'ai embrassé la main de sa Majesté ${ }^{53}$.

\footnotetext{
${ }^{48}$ A. Fanshawe, The Memoirs..., op. cit., p. 133-134. «[...] upon the 2 day of September following was fought the Battle of Woster [Worcester], when the King being missed and nothing of your father being dead or alive for 3 days heard of [...] I neither eat nor sleep, but trembled at every motion I heard, expecting the fatal news which at last came in their newsbook, which mentioned your father a prisoner. »

${ }^{49}$ A. Fanshawe, The Memoirs..., op. cit., p. 119: «my life and necessity, not delight or revenge, hath made insert some passages which will reflect on their owners, as the praises of others will be but just, which is my intent in this narrative. »

${ }^{50}$ A. Fanshawe, The Memoirs..., op. cit., p. 132. Sur les relations avec Hyde et sur la critique de Charles II, voir Madeleine Bassnet, "All the ceremonyes and civilityes": The Authorship of Diplomacy in the Memoirs of Ann, Lady Fanshawe », The Seventeenth Century, vol. 26, n 1, 2011, pp. 94-118.

${ }^{51}$ A. Halkett, The Memoirs..., op. cit., p. 26.

52 A. Halkett, The Memoirs..., op. cit., p. 61: "How comes (said I) you have taken the power from the Parliamentt and those successive interests that have governed since you wanted the King?" / "Because, said hee, wee found after a little time they began to bee as bad as hee, and therfore wee changed". » Sur cette transcription des discours prononcés, voir E. Lesne, La Poétique des Mémoires, op. cit., p. 211.

53 Ibid., pp. 53-54 : «[The King] came to mee and said, 'Mrs. Murray, I am ashamed I have been so long a'speaking to you, butt itt was because I could nott say enough to you for the service you did my brother. Butt if
} 
Cependant, malgré le culte qu'elle voue au roi, la mémorialiste, en d'autres endroits, s'emploie, comme Margaret Cavendish et Ann Fanshawe à démystifier la cour et ses factions. Ainsi, par exemple, elle évoque le destin tragique de son jeune frère William «très injustement banni de trois cours, celle du roi, celle de la Reine mère, et celle de Princesse royale ${ }^{54} »$, parce qu'il était suspecté d'avoir voulu faire couronner le duc d'York avec la complicité du colonel Bampfield :

Vers cette époque, mon frère Will rentra très mécontent, et pour de bonnes raisons, car des personnes s'étaient employées à jeter des semences de jalousie entre le roi et le duc d'York ; à la suite de quoi, ils accusèrent mon frère d'entretenir une correspondance avec C. B., qui entretenait depuis Londres des relations secrètes avec l'Écosse, et leur dessein était de faire couronner dans cette contrée le duc d'York $^{55}$.

Ce passage, comme beaucoup d'autres, montre qu'on a eu tort de trop vouloir « dépolitiser » les Mémoires de Lady Anne Halkett ; on voit ici au contraire que la mémorialiste, comme ses contemporaines, se place sur le terrain de la politique et de l'histoire ${ }^{56}$. De ce point de vue le colonel Bampfield n'est pas seulement un personnage romanesque peu scrupuleux, mais aussi et surtout une figure historique dont il faut à tout prix révéler la traîtrise à l'égard de la monarchie.

Le bref examen de la poétique des Mémoires de Margaret Cavendish, d'Anne Halkett et d'Ann Fanshawe permet de corriger l'idée que l'histoire de la Révolution anglaise aurait seulement été écrite par des hommes. La fluidité entre les sexes qui caractérise la vision du genre à l'époque moderne se vérifie aussi dans l'historiographie. À l'instar des hommes, les femmes se présentent comme des témoins avertis de l'histoire, et optent, comme eux, pour le genre des Mémoires, un genre qui les conduit à relire leur expérience de la Révolution et à corriger l'histoire officielle. Selon nous, ces versions féminines de l'histoire méritent d'être entendues et intégrées dans l'histoire nationale de la Révolution anglaise, une histoire mixte $^{57}$, nécessairement complexe et polyphonique qui, comme le souligne l'historien Thomas May dès 1647, ne peut s'écrire qu'à plusieurs mains :

Le monde a su par la renommée combien d'actes de valeur la nation anglaise, dans les deux partis, avait eu à se reprocher dans cette lutte dénaturée. Mais on ne saura jamais parfaitement tout ce qu'ont montré de vertu, de mérite et de courage, tels ou tels lords, gentilhommes ou autres, à moins que ces choses ne soient rapportées par les écrivains des deux partis ${ }^{58}$.

\section{Claire GHEERAERT-GRAFFEUILLE, Université de Rouen, ERIAC}

\footnotetext{
ever I can command what I have right to as my owne, there shall bee nothing in my power I will not do for you. And with that the King laid his hand upon both mine as they lay upon my breast. I humbly bowed down and kist his Majesty's hand. »

${ }^{54}$ Ibid. p. 29 : « This account I had from Mr. N, who was [...] witnesse of it. »

${ }^{55}$ Ibid., p. 28: «Aboutt this time my brother Will came home much discontented, as hee had great reason, for some persons, who made itt there busynese to sow the seed of jelousye betwixt the King and Duke of Yorke, in pursuite of that accused my brother that hee kept a correspondence with C. B., who staid att London to hold intelligence in Scotland, and ther designe was to have the Duke of Yorke come there to bee crowned King. »

${ }^{56}$ S. Wiseman, Conspiracy and Virtue, op. cit., p. 326.

${ }^{57}$ Sur l'idée d'une histoire mixte, voir Sophie Vergnes, « Des discours de la discorde : les femmes, la Fronde et l'écriture de l'histoire », Études Épistémè, 2011, n¹9.

${ }^{58}$ Voir Thomas May, Histoire du Long-Parlement convoqué par Charles $I^{e r}$ en 1640, Paris, chez Béchet Ainé, 1823, p. 6: «How much valour the English Nation on both sides have been guilty of in this unnaturall Warre, the World must needs know in the generall fame. But for particulars, how much Worth, Vertue, and Courage, some particular Lords, Gentlemen, and others have shewed, unlesse both sides do write, will never perfectly be known » (T. May, The History of the Parliament of England, London, 1647, op. cit., sig. B2).
} 